\title{
Free Serum Testosterone Versus Total Testosterone/Estradiol Ratio in Low Sexual Desire in Old Men
}

\author{
Ahmed SA Abouroab*1, Hamdy Foad Aly Marzok ${ }^{2}$, Sherif Refaat Ismail ${ }^{1}$ \\ Departments of ${ }^{1}$ Andrology \& STDs and ${ }^{2}$ Clinical Pathology, Faculty of Medicine, \\ Mansoura University. Mansoura, Egypt \\ *Corresponding author: Ahmed SA Abouroab, Mobile: (+20) 01062620606, E-Mail: dr.ahmedsami2010@ yahoo.con
}

\begin{abstract}
Background: Testosterone (T) and estradiol (E2) play a critical role in male sexual function being essential for modulating libido and erectile function. We hypothesized that an imbalance of $\mathrm{T} / \mathrm{E} 2$ ratio may be an important factor leading to the development of low sexual desire in ageing men.

Objective: To evaluate the relationship between imbalance of T/E2 ratio and low sexual desire in ageing men.

Patients and Methods: This study was conducted on 50 low sexual desire old men and 40 healthy control men (mean age 59.8 years \& range 55-69 years). These men were subjected to history taking, clinical examination, sexual desire score of IIEF and SDI-2 as well as estimation of serum sex hormones (total T, free T, E2, prolactin, and SBHG) using ELISA method.

Results: The sexual desire score of IIEF and SDI-2 were significantly lower in patients compared with the controls. The levels of TT, E2, TT/E2, FT and FT/E2 were significantly lower among study group compared with the controls. On the other hand, there were significant positive correlations between FT and FT/E2 ratio and both sexual desire score of IIEF and SDI-2. SHBG, E2, TT/E2 showed poor AUCs (AUC $=0.651,0.626,0.685$ respectively). FT/E2 showed fair AUC $(A U C=0.727)$. Serum FT showed good AUC $(A U C=0.882)$, TT, combined T/E2 and FT and combined TT and E2 showed excellent AUC (AUC $=0.900,0.903,0.958$ respectively).

Conclusion: Although older men with low sexual desire had significantly lower total and free T, our study failed to show significant correlations between total T, E2 or TT/E2 ratio and sexual desire score of IIEF and SDI-2. However, true androgen deficiency (FT) and true T/E2 imbalance (FT/E2 ratio) may have a role in discrimination between low and normal sexual desire in older men.
\end{abstract}

Keywords: Testosterone, Estradiol ratio, Sexual desire, Men, Elderly.

\section{INTRODUCTION}

Sexual desire is a motivational state and an interest in "sexual objects or activities, or as a wish, or drive to seek out sexual objects or to engage in sexual activities" (1). Human beings are actually never too old to enjoy a happy and healthy sex life. Despite this, many people, young and old alike, are astounded at the idea of people remaining sexually active in their sixties and beyond ${ }^{(2)}$. The importance of androgens in establishing and maintaining sexual function in males of most species is well recognized. Estrogens also stimulate male sexual function in some species ${ }^{(3)}$.

It is now well accepted that serum testosterone (T) levels decline progressively with aging in men ${ }^{(4)}$. Circulating $\mathrm{T}$ is approximately $98 \%$ bound to serum proteins, predominantly to sex hormone-binding globulin (SHBG), the major binding protein for $\mathrm{T}$ in blood, and albumin ${ }^{(5)}$. Only $1 \%$ to $2 \%$ of $\mathrm{T}$ in circulation is completely unbound or free. Because $\mathrm{T}$ is bound with high affinity to SHBG, SHBG-bound T is not available to most tissues for action. In contrast, $\mathrm{T}$ is bound with low affinity to albumin, so both albumin-bound and free $\mathrm{T}$ are bioavailable to most tissues for action ${ }^{(6)}$.

The concentration of SHBG increases with aging, serum-free $\mathrm{T}$ and bioavailable $\mathrm{T}$ (free plus albumin-bound $\mathrm{T}$ ) concentrations decline markedly than total $\mathrm{T}$ levels with aging ${ }^{(7)}$. It has been demonstrated that the descent in testosterone levels is one of the best known hormonal factors affecting sexual function, and $\mathrm{T}$ is deeply involved in every step of the male sexual response ${ }^{(8)}$. Additionally, patients with low levels of testosterone may have decreased libido ${ }^{(9)}$. In aging males, estrogen plays important roles in the regulation of gonadotropin production, the emotional state, bone mass and lipid synthesis ${ }^{\left({ }^{(10)}\right.}$. In normal persons, there is a balance between $\mathrm{T}$ and $\mathrm{E} 2$ with $\mathrm{T} / \mathrm{E} 2$ ratio $=10^{(\mathbf{1 1})}$.

We hypothesized that an imbalance of T/E2 ratio may be an important factor leading to the development of low sexual desire in ageing men. Owing to these data, we consider that it may not be enough to investigate the association between serum $T$ and low sexual desire in ageing men. Therefore, we might combine estradiol and T together, i.e. T/E2 ratio, to further study the relationship between sex hormones and low sexual desire in ageing men.

In this study, we measured the levels of the serum sex hormones (total T, free $\mathrm{T}$ and E2) in both normal controls and old men complaining of low sexual desire, and observed whether the T or free T/E2 ratio is imbalanced in cases of low sexual desire in ageing men, and then to explore further the relationship between imbalanced sex hormones and low sexual desire in ageing men. 


\section{PATIENTS AND METHODS}

This prospective hospital-based cross-sectional study has been carried on 50 patients $>55$ years with low sexual desire and 40 control subjects with normal desire for their age. Patients have been recruited from Andrology Outpatient Clinic, University Hospital during the period between Aug-Dec 2019. The patients were classified into two groups; Group A: older individuals with low sexual desire and group B: older individuals with normal sexual desire. After obtaining written informed consents the participants had been enrolled in this study.

Inclusion criteria: Old Patients > 55 years with low sexual desire (Patients with questions 11 and 12 of IIEF-15 scores between 1 and 5), married patients and sexual activity in the last 6 months.

Exclusion criteria: Patient $<55$ years with low sexual desire, patients $>55$ year with normal sexual desire (with questions 11 and 12 of IIEF-15 scores between with 6 to 10), patients $>55$ year with low sexual desire but suffer from chronic diseases as diabetes mellitus, hypertension, endocrinal disorders, chronic debilitating diseases, psychiatric disorders or malignancies or use of medications that could affect hormone levels (antihypertensives, antidepressants, antiandrogens, narcotics, analgesics, anesthetics, sedatives, tranquilizers phencyclidine, and alcohol).

These participants were subjected to the following:

- History taking (onset, duration, previous treatments, and special habits).

- Clinical examination for exclusion of possible conditions which can affect sexual desire.

- Questionnaire to assess the sexual desire (SDI-2) ${ }^{(\mathbf{1 2})}$.
- Assessment of serum levels of fasting samples of total testosterone, free testosterone, estradiol, prolactin, and sex hormone binding globulin by the enzyme-linked immunosorbent assay (ELISA) method (ATLAS Medical, William James House, Cowley Road, Cambridge, CB4 0WX, UK). T/E2 ratio was calculated by the formula (Testosterone $/ 10^{*}$ Estradiol) ${ }^{(13)}$.

\section{Ethical approval:}

An approval of the study was obtained from Mansoura University academic and ethical committee. Every patient signed an informed written consent for acceptance of the operation.

\section{Statistical Analysis:}

The results of was tabulated and statistically analyzed with SPSS analysis software version 23 (SPSS Inc., Chicago, IL, U.S.A.). All parameters were subjected to test the normality of data distribution. Parametric numerical data was presented as a mean \pm Standard $(\mathrm{x} \pm \mathrm{SD})$ and was analysed using statistical parametric tests. Nonparametric numerical data was presented as median and range and was analyzed using non-parametric tests. Statistical significance was established at $\mathrm{p} \leq$ 0.05 .

\section{RESULTS}

Table (1) showed the differences between clinical and laboratory variables among control and study groups. The serum levels of TT, E2, FT and TT/E2 as well as FT/E2 ratios were significantly lower among study group compared with the controls.

Table (1): Differences between clinical and laboratory variables among control and study groups

\begin{tabular}{|l|c|c|c|c|c|}
\hline \multicolumn{2}{|l|}{} & $\begin{array}{c}\text { Controls } \\
\mathbf{N = 4 0}\end{array}$ & $\begin{array}{c}\text { Study group } \\
\mathbf{N = 5 0}\end{array}$ & Test value• & P-value \\
\hline Age (year) & Mean \pm SD & $58.4 \pm 3.2$ & $59.8 \pm 4.3$ & $\mathrm{~T}=-1.480$ & 0.177 \\
\hline $\begin{array}{l}\text { Desire disorder } \\
\text { duration (months) }\end{array}$ & Mean \pm SD & ----- & $7.8 \pm 2.2$ & $-\cdots--$ & ------ \\
\hline $\begin{array}{l}\text { IIEF score } \\
\text { (maximum score=) }\end{array}$ & Mean \pm SD & $36.2 \pm 2.1$ & $23.5 \pm 1.5$ & $\mathrm{~T}=31.548$ & $<\mathbf{0 . 0 0 1}$ \\
\hline $\begin{array}{l}\text { Sexual desire score } \\
\text { of IIEF } \\
\text { (maximum score=) }\end{array}$ & Mean \pm SD & $6.4 \pm 0.6$ & $3.9 \pm 0.8$ & $\mathrm{~T}=16.703$ & $<\mathbf{0 . 0 0 1}$ \\
\hline $\begin{array}{l}\text { SDI-2 } \\
\text { (maximum score=) }\end{array}$ & Mean \pm SD & $48.0 \pm 1.8$ & $39.4 \pm 2.5$ & $\mathrm{~T}=18.719$ & $<\mathbf{0 . 0 0 1}$ \\
\hline Prolactin (ng/ml) & Mean \pm SD & $14.6 \pm 4.2$ & $18.7 \pm 6$ & $\mathrm{~T}=-2.519$ & $\mathbf{0 . 0 0 7}$ \\
\hline SHBG (nmol/L) & Mean \pm SD & $28.7 \pm 6.3$ & $35 \pm 1.1$ & $\mathrm{~T}=-2.462$ & $<\mathbf{0 . 0 0 1}$ \\
\hline TT (ng/ml) & Mean \pm SD & $5.5 \pm 1$ & $3.8 \pm 0.9$ & $\mathrm{~T}=9.380$ & $<\mathbf{0 . 0 0 1}$ \\
\hline E2 (pg/ml) & Mean \pm SD & $20.9 \pm 6.6$ & $18.1 \pm 5.6$ & $\mathrm{~T}=1.971$ & $\mathbf{0 . 0 3 0}$ \\
\hline TT/E2 & Mean \pm SD & $29.9 \pm 5$ & $22.9 \pm 4.3$ & $\mathrm{~T}=3.551$ & $\mathbf{0 . 0 0 2}$ \\
\hline FT (pg/ml) & Mean \pm SD & $19.6 \pm 4.1$ & $12.2 \pm 2.5$ & $\mathrm{~T}=5.914$ & $<\mathbf{0 . 0 0 1}$ \\
\hline FT/E2 & Mean \pm SD & $1 \pm 0.3$ & $0.7 \pm 0.2$ & $\mathrm{~T}=3.821$ & $<\mathbf{0 . 0 0 1}$ \\
\hline
\end{tabular}

SHBG, sex hormone-binding globulin;

TT, Total testosterone; FT, Free testosterone; E2, Estradiol.

Both serum FT and FT/E2 ratio were significantly lower among severe low sexual desire patients compared to those with mild to moderate severity (Table 2). 
Table (2): Comparison of laboratory data between severe and mild to moderate sexual desire disorder cases

\begin{tabular}{|c|c|c|c|c|c|c|c|}
\hline \multirow{3}{*}{ Prolactin (ng/ml) } & \multirow{3}{*}{$\begin{array}{c}\text { Median } \\
\text { Min-max }\end{array}$} & \multirow{2}{*}{\multicolumn{2}{|c|}{$\begin{array}{c}\text { Mild to moderate cases } \\
\mathbf{N = 3 6 , \text { Score 4-5 }} \\
16\end{array}$}} & \multirow{2}{*}{\multicolumn{2}{|c|}{$\begin{array}{c}\begin{array}{c}\text { Severe cases } \\
\mathbf{N}=\mathbf{1 4}, \text { Score }=\mathbf{2 - 3}\end{array} \\
13 \\
\end{array}$}} & \multirow{3}{*}{$\begin{array}{l}\text { Test -value } \\
Z=1.593\end{array}$} & \multirow{3}{*}{$\frac{\mathbf{p}}{0.118}$} \\
\hline & & & & & & & \\
\hline & & 8 & 42 & 9 & 31 & & \\
\hline SHBG (nmol/L) & Mean \pm SD & 34.9 & 9.9 & 35.2 & 11 & $\mathrm{~T}=-0.092$ & 0.927 \\
\hline TT (ng/ml) & Mean \pm SD & 3.8 & 0.9 & 3.9 & 0.8 & $\mathrm{~T}=-0.566$ & 0.574 \\
\hline E2 $(\mathrm{pg} / \mathrm{ml})$ & Mean \pm SD & 18.4 & 6.1 & 17.3 & 3.5 & $\mathrm{~T}=0.837$ & 0.407 \\
\hline \multirow{2}{*}{ TT/E2 } & Median & \multicolumn{2}{|c|}{20.2} & \multicolumn{2}{|c|}{22.5} & \multirow{2}{*}{$\mathrm{T}=-0.310$} & \multirow{2}{*}{0.758} \\
\hline & Min-max & 8.5 & 48.1 & 11.5 & 37.2 & & \\
\hline FT $(\mathrm{pg} / \mathrm{ml})$ & Mean \pm SD & 13.6 & 4.4 & 8.6 & 2 & $\mathrm{~T}=5.506$ & $<0.001$ \\
\hline FT/E2 & Mean \pm SD & 0.8 & 0.2 & 0.5 & 0.1 & $\mathrm{~T}=2.842$ & 0.007 \\
\hline
\end{tabular}

SHBG, sex hormone-binding globulin; $\quad$ TT, total testosterone; FT, free testosterone; E2, estradiol.

Mild to moderate cases: sexual desire score of questions 11 and 12 of IIEF 4-5. Severe: sexual desire score of questions 11 and 12 of IIEF 2-3.Regarding correlation matrix, FT and FT/E2 showed significantly positive correlation with IIEF, sexual desire and SDI-2 (Table 3).

Table (3): Correlations analyses between hormones and other clinical and laboratory parameters

\begin{tabular}{|c|c|c|c|c|c|c|c|c|c|c|c|c|}
\hline \multirow[b]{2}{*}{$\begin{array}{l}\text { Study } \\
\text { group }\end{array}$} & \multicolumn{2}{|c|}{ SHBG } & \multicolumn{2}{|c|}{ TT } & \multicolumn{2}{|c|}{ E2 } & \multicolumn{2}{|c|}{ T/E2 } & \multicolumn{2}{|c|}{ FT } & \multicolumn{2}{|c|}{ FT/E2 } \\
\hline & $\mathbf{r}$ & p & $\mathbf{r}$ & $\mathbf{p}$ & $\mathbf{r}$ & $\mathbf{p}$ & $\mathbf{r}$ & $\mathbf{p}$ & $\mathbf{r}$ & $\mathbf{p}$ & $\mathbf{r}$ & $\mathbf{p}$ \\
\hline Age & $0 . \overline{068}$ & 0.637 & 0.001 & 0.993 & $0 . \overline{155}$ & 0.281 & 0.163 & 0.258 & $0 . \overline{030}$ & 0.836 & 0.084 & 0.563 \\
\hline IIEF & 0.131 & 0.365 & 0.128 & 0.377 & 0.055 & 0.705 & 0.001 & 0.995 & 0.692 & $<0.001$ & 0.429 & 0.002 \\
\hline $\begin{array}{c}\text { Sexual } \\
\text { desire }\end{array}$ & $\begin{array}{c}- \\
0.016 \\
\end{array}$ & 0.911 & $\begin{array}{c}- \\
0.108 \\
\end{array}$ & 0.453 & 0.035 & 0.811 & $\begin{array}{c}- \\
0.152 \\
\end{array}$ & 0.293 & 0.516 & $<0.001$ & 0.345 & 0.014 \\
\hline SDI-2 & 0.024 & 0.871 & 0.071 & 0.624 & $0 . \overline{112}$ & 0.441 & 0.054 & 0.709 & 0.588 & $<0.001$ & 0.482 & $<0.001$ \\
\hline Prolactin & $\begin{array}{c}- \\
0.122 \\
\end{array}$ & 0.400 & $\begin{array}{c}- \\
0.122 \\
\end{array}$ & 0.398 & 0.226 & 0.115 & $0 . \overline{245}$ & 0.087 & 0.137 & 0.342 & -0.038 & 0.792 \\
\hline SHBG & - & - & 0.034 & 0.815 & $\begin{array}{c}- \\
0.205 \\
\end{array}$ & 0.152 & 0.151 & 0.296 & 0.208 & 0.147 & 0.226 & 0.115 \\
\hline TT & - & - & - & - & $\begin{array}{c}- \\
0.091\end{array}$ & 0.528 & 0.679 & $<0.001$ & 0.187 & 0.192 & 0.155 & 0.283 \\
\hline E2 & - & - & - & - & - & - & $\begin{array}{c}- \\
0.756 \\
\end{array}$ & $<0.001$ & $0 . \overline{0} 6$ & 0.856 & -0.565 & $<0.001$ \\
\hline TT/E2 & - & - & - & - & - & - & - & - & 0.099 & 0.493 & 0.540 & $<0.001$ \\
\hline FT & - & - & - & - & - & - & - & - & - & - & 0.735 & $<0.001$ \\
\hline $\begin{array}{c}\text { Control } \\
\text { group }\end{array}$ & $\mathbf{r}$ & $\mathbf{p}$ & $\mathbf{r}$ & $\mathbf{p}$ & $\mathbf{r}$ & $\mathbf{p}$ & $\mathbf{r}$ & $\mathbf{p}$ & $\mathbf{r}$ & $\mathbf{p}$ & $\mathbf{r}$ & $\mathbf{p}$ \\
\hline Age & 0.131 & 0.420 & 0.184 & 0.254 & 0.036 & 0.823 & 0.095 & 0.559 & 0.081 & 0.619 & -0.043 & 0.793 \\
\hline IIEF & 0.129 & 0.429 & $0 . \overline{-} 35$ & 0.144 & 0.247 & 0.125 & $\begin{array}{c}- \\
0.228 \\
\end{array}$ & 0.157 & 0.393 & 0.012 & 0.222 & 0.169 \\
\hline SDI-2 & 0.466 & 0.002 & 0.096 & 0.554 & $\begin{array}{c}- \\
0.032 \\
\end{array}$ & 0.842 & 0.078 & 0.630 & 0.383 & 0.015 & 0.471 & 0.002 \\
\hline Prolactin & 0.435 & 0.005 & 0.153 & 0.345 & 0.078 & 0.634 & 0.037 & 0.822 & 0.016 & 0.922 & 0.338 & 0.033 \\
\hline SHBG & - & - & 0.240 & 0.135 & $\overline{0} \cdot \overline{115}$ & 0.482 & 0.234 & 0.146 & 0.076 & 0.641 & -0.062 & 0.702 \\
\hline TT & - & - & - & - & $\begin{array}{c}- \\
0.504 \\
\end{array}$ & 0.001 & 0.737 & $<0.001$ & $\begin{array}{c}- \\
0.305 \\
\end{array}$ & 0.056 & 0.177 & 0.276 \\
\hline E2 & - & - & - & - & - & - & $\begin{array}{c}- \\
0.923 \\
\end{array}$ & $<0.001$ & 0.164 & 0.312 & -0.534 & $<0.001$ \\
\hline TT/E2 & - & - & - & - & - & - & - & - & $0 . \overline{2} 46$ & 0.126 & 0.533 & $<0.001$ \\
\hline FT & - & - & - & - & - & - & - & - & - & - & 0.616 & $<0.001$ \\
\hline
\end{tabular}

Pearson's correlation, ROC curve analysis of SHBG, TT, E2, TT/E2 and FT levels was conducted for discrimination between low and normal sexual desire old men groups (Table 4). SHBG, E2, TT/E2 showed poor AUCs (AUC = $0.651,0.626,0.685$ respectively). FT/E2 showed fair AUC $($ AUC $=0.727)$. Serum FT showed good AUC $($ AUC $=$ 0.882), TT, combined T/E2 \& FT and combined TT \& E2 showed excellent AUC (AUC $=0.900,0.903,0.958$ ) respectively. 
Table (4): Validity of hormonal levels for discrimination between different studied groups

\begin{tabular}{|c|c|c|c|c|c|c|c|c|}
\hline Biomarker & SHBG & TT & E2 & TT/E2 & FT & FT/E2 & $\begin{array}{c}\text { Combined } \\
\text { T/E2 and } \\
\text { FT }\end{array}$ & $\begin{array}{c}\text { Combined } \\
\text { TT and E2 }\end{array}$ \\
\hline AUC & 0.651 & 0.9 & 0.626 & 0.685 & 0.882 & 0.727 & 0.903 & 0.958 \\
\hline (CI 95\%) & $0.842-$ & $0.517-$ & $0.579-$ & $0.814-$ & $0.814-$ & $0623-$ & $0.840-$ & $0.923-$ \\
& 0.958 & 0.735 & 0.791 & 0.950 & 0.950 & 0.831 & 0.966 & 0.992 \\
\hline P value & 0.029 & $<0.001$ & 0.068 & 0.001 & $<0.001$ & $<0.001$ & $<0.001$ & $<0.001$ \\
\hline Cut off & 36 & 4.65 & 18.95 & 27.7 & 16.65 & 0.94 & $27.7+16.65$ & $4.65+18.95$ \\
\hline $\begin{array}{c}\text { Sensitivity } \\
(\%)\end{array}$ & 46 & 78 & 66 & 78 & 80 & 80 & 78 & 86 \\
\hline $\begin{array}{c}\text { Specificity } \\
(\%)\end{array}$ & 90 & 86 & 60 & 66 & 86 & 60 & 92.5 & 92.5 \\
\hline PPV (\%) & 82.1 & 84.8 & 62.3 & 69.6 & 85.1 & 71.4 & 92.9 & 93.5 \\
\hline $\begin{array}{c}\text { NPV (\%) } \\
\text { (\%) }\end{array}$ & 62.5 & 79.6 & 63.8 & 75 & 81.1 & 70.6 & 77.1 & 84.1 \\
\hline $\begin{array}{c}\text { Accuracy } \\
\text { (\%) }\end{array}$ & 68 & 82 & 63 & 72 & 83 & 71.1 & 84.4 & 88.9 \\
\hline
\end{tabular}

AUC, area under receiver operating curve (ROC); PPV, positive predictive value;

NPV, negative predictive value.

\section{DISCUSSION}

Our study showed that the studied old men with low sexual desire had significantly lower total and free testosterone levels when compared to old men with normal sexual desire. Our results are in agreement with Zitzmann et al. ${ }^{(13)}$ who noted that low blood testosterone levels were associated with increased prevalence of syndromes such as sexual dysfunction, sexual disinclination, and obesity, lack of concentration, somnipathy, and erectile dysfunction (ED) in a large series of 434 males aged 50-86 years. Furthermore, Ahn et al. ${ }^{(14)}$ showed that testosterone was lower in elderly than in young men. Moreover, other studies by Araujo et al. (15) and Caronia et al. ${ }^{(16)}$ have assessed the changes in testosterone and associated reproductive hormones during adult life in men, and showed that in healthy men the mean serum TT levels decrease by $<30 \%$ from 25 to 75 years of age, whereas mean serum free testosterone levels decrease by as much as half over the same period. In addition, Cunningham et al. ${ }^{(17)}$ found that testosterone was lower in elderly than in young men. On the other hand, the relationship of testosterone levels with sexual activity in older men showed controversial results in some studies ${ }^{(18,19}$, and 20).

The IIEF was designed to measure erection ability and to evaluate a medical effect of ED. In our study, the sexual desire score of IIEF and the sexual desire inventory (SDI-2 were significantly lower in cases compared to the control group. The SDI-2 is the most tested instrument about sexual

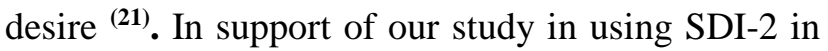
old men, Yee $\boldsymbol{e t}$ al. ${ }^{(22)}$ study showed normal healthy men with significantly reported higher SDI-2 scores as compared to individuals with benign prostate hyperplasia (BPH) with the conclusion that SDI-2 can be used as a reliable and valid tool in assessing sexual desire among men. Additionally, Cunningham et al. (17) analyzed correlations between blood total testosterone value and scores related to sexual function syndromes in hospital visitors with hyposexuality such as ED and sexual disinclination and found that baseline FT and TT, but not E2 or SHBG, were consistently and independently associated with measures of sexual desire, erectile function and sexual activity in older men with low testosterone levels. They concluded that the circulating FT and TT levels contribute more than E2 or SHBG to the variation in some measures of sexual dysfunction in older men with low TT.

Our study showed statistically significant difference between the two groups regarding E2 $(\mathrm{pg} / \mathrm{ml})$. Besides, there was highly statistically significant difference found between the two groups regarding prolactin $(\mathrm{ng} / \mathrm{ml})$, SHBG $(\mathrm{nmol} / \mathrm{L})$, TT $(\mathrm{ng} / \mathrm{ml})$, TT/E2, FT $(\mathrm{pg} / \mathrm{ml})$ and FT/E2. These findings may suggest that these hormonal disturbances may be responsible for the low sexual desire in our patients. In support of our results, it is well accepted that there is a gradual, age-related decline in serum total and free testosterone levels in healthy adult men ${ }^{(23)}$. Moreover, cross-sectional studies have also demonstrated a significant increase in sex hormone-binding globulin (SHBG) concentrations in the aging male. Thus, not only does total testosterone decline, but also a higher percentage of the remaining testosterone is bound tightly to SHBG, further reducing the amount of bioavailable

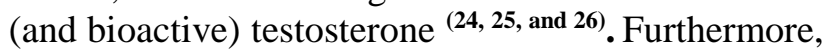
other data demonstrated that the free testosterone levels at age of 75 years are $50 \%$ as those found in men at age 25. However, not all aged men have abnormal free testosterone levels, even though the levels may be half those of men much younger. While there is little debate on how to define hypogonadism 
in the young man, controversy still exists regarding the definition of hypogonadism in the aged individuals ${ }^{(27)}$.

Our study failed to show significant correlations between total T, E2 or TT/E2 ratio and sexual desire score of IIEF and SDI-2, respectively. On the other hand, there were significant positive correlations between FT and FT/E2 ratio and both sexual desire score of IIEF and SDI-2 suggesting a role of the active fragment of $\mathrm{T}$ in the sexual desire. In agreement with our results, Castelló-Porcar and Martínez-Jabaloyas (28) observed that FT and bioavailable $\mathrm{T}$ were the only variables that showed positive linear association with ED and decreased sexual desire. However, with regard to sexual desire, the TT value was not correlated and many patients had normal sexual desire. Actually, it has been reported that the numerical $\mathrm{T}$ value, which is required to preserve sexual desire is somewhat low. In contrast, others have reported that the numerical value of $\mathrm{T}$ was not largely associated with sexual desire, although others reported a correlation between the numerical value of $\mathrm{T}$ and sexual desire ${ }^{(29,30)}$.

The role of estrogens in sexually reproductive males is not well defined. In the study by O'Connor et $\boldsymbol{a l} .{ }^{(31)}$ an association between both testosterone levels and ED, as well as with the frequency of masturbation was observed, but this association was not demonstrated with estradiol.

TT/E2 showed significantly positive correlation with TT and significantly negative correlation with E2. FT showed significantly positive correlation with IIEF, sexual desire score of IIEF and SDI-2. ; FT/E2 showed significantly positive correlation with IIEF, sexual desire, SDI-2, TT/E2 and FT, while significantly negative correlation with E2. These finding may suggest a role for FT/E2 balance in sexual desire function in old men. The role of E2 received attention from Castelló-Porcar and Martínez-Jabaloyas ${ }^{(28)}$. The authors have concluded that even after adjusting for $\mathrm{T}$ levels, it was observed that men with higher levels of estradiol had more discomfort in their sexual function, two different mechanisms of action in sexual function could have an effect: T levels that influence behavior, and E2 levels that decreases the sexual function associated with a depressive state. The role for FT/E2 imbalance in sexual desire function in old men appears to be more important than E2 levels alone in old men. Thus, with increasing age, testosterone aromatization to estradiol, along with low testosterone levels, could be one of the causes of abnormal sexual function in men (11, 28). Additionally, Chen et al. ${ }^{(32)}$ also found no association between the T/E ratio and the severity of ED and decreased libido. Moreover, Tan et al. ${ }^{(33)}$ conducted a study including 34.016 patients who were treated from hyperestrogenism using exogenous testosterone and with levels of hyperestrogenism.
They found that there was no association between high estradiol levels and decreased sexual desire. To the contrary, decreased libido was associated with normal and low estradiol levels.

ROC curve analysis of SHBG, TT, E2, TT/E2 and FT levels was conducted for discrimination between low and normal sexual desire old men groups. SHBG, E2, TT/E2 showed poor AUCs (AUC $=0.651,0.626,0.685$ respectively). FT/E2 showed fair AUC (AUC $=0.727)$. Serum FT showed good AUC (AUC $=0.882$ ). TT, combined T/E2 \& FT and combined TT \& E2 showed excellent AUC (AUC = $0.900,0.903,0.958$ respectively). These findings may suggest that true androgen deficiency (FT) and true T/E2 imbalance (FT/E2 ratio) may have a role in discrimination between low and normal sexual desire in old men.

This study has some limitations, one of them is the low number of the participants, TT and TT/E2 were selected as the main hormone parameters of this study, which is not proportional to free testosterone in some cases.

\section{CONCLUSION}

Although older men with low sexual desire had significantly lower total and free $\mathrm{T}$, our study failed to show significant correlations between total T, E2 or TT/E2 ratio and sexual desire score of IIEF and SDI-2. However, true androgen deficiency (FT) and true T/E2 imbalance (FT/E2 ratio) may have a role in discrimination between low and normal sexual desire in older men.

\section{REFERENCES:}

1. Regan P, Atkins L (2006): Sex differences and similarities in frequency and intensity of sexual desire. Social Behavior and Personality: An International Journal, 34 (1): 95-102.

2. Pfeiffer E, Verwoerdt A, Davis G (1972): Sexual behavior in middle life. American Journal of Psychiatry, 128 (10): 1262-1267.

3. Bagatell C, Heiman J, Rivier J et al. (1994): Effects of endogenous testosterone and estradiol on sexual behavior in normal young men. The Journal of Clinical Endocrinology \& Metabolism, 78 (3): 711-716.

4. Matsumoto A (1993): 'Andropause': Are reduced androgen levels in aging men physiologically important? Western Journal of Medicine, 159 (5): 618-23.

5. Dunn J, Nisula B, Rodbard D (1981): Transport of steroid hormones: binding of 21 endogenous steroids to both testosterone-binding globulin and corticosteroidbinding globulin in human plasma. The Journal of Clinical Endocrinology \& Metabolism, 53 (1): 58-68.

6. Nahoul K, Roger M (1990): Age-related decline of plasma bioavailable testosterone in adult men. Journal of Steroid Biochemistry, 35 (2): 293-299.

7. Feldman H, Longcope C, Derby C et al. (2010): Age trends in the level of serum testosterone and other hormones in middle-aged men: longitudinal results from the Massachusetts Male Aging Study. J Clin Endocrinol Metab., 95: 4542-8. 
8. Rastrelli G, Corona G, Maggi M (2018): Testosterone and sexual function in men. Maturitas, 112: 46-52.

9. Vermeulen A, Kaufman J, Pottelberg I et al. (2002): Estradiol in elderly men. Aging Male, 5: 98-102.

10. Schulster M, Bernie A, Ramasamy R (2016): The role of estradiol in male reproductive function. Asian $\mathbf{J}$ Androl., 18 (3): 435-40. structure, and evidence of reliability. Journal of Sex \& Marital Therapy, 22 (3): 175-90.

11. Spector I, Carey M, Steinberg L (1996): The sexual desire inventory: Development, factor structure, and evidence of reliability. Journal of Sex \& Marital Therapy, 22 (3): 175-90.

12. Zakharov M, Bhasin S, Travison T et al. (2015): A multistep, dynamic allosteric model of testosterone's binding to sex hormone binding globulin. Mol Cell Endocrinol., 399: 190-200.

13. Zitzmann M, Faber S, Nieschlag E (2006): Association of specific symptoms and metabolic risks with serum testosterone in older men. J Clin Endocrinol Metab., 91:4335-4343.

14. Ahn H, Park C, Lee $S$ (2002): The clinical relevance of sex hormone levels and sexual activity in the ageing male. BJU International, 89: 526-530.

15. Araujo A, Wittert G (2011): Endocrinology of the aging male. Best Pract Res Clin Endocrinol Metab., 25 (2): 303 319.

16. Caronia L, Dwyer A, Hayden D et al. (2012): Abrupt Decrease in Serum Testosterone Levels After an Oral Glucose Load in Men: Implications for Screening for Hypogonadism. Clin Endocrinol (Oxf), 78 (2): 291-6.

17. Cunningham G, Stephens-Shields A, Rosen $\mathrm{R}$ et al. (2015): Association of sex hormones with sexual function, vitality, and physical function of symptomatic older men with low testosterone levels at baseline in the testosterone trials. J Clin Endocrinol Metab., 100 (3): 1146-1155.

18. Gades N, Jacobson D, McGree M et al. (2008): The associations between serum sex hormones, erectile function, and sex drive: the Olmsted County Study of Urinary Symptoms and Health Status among Men. J Sex Med., 5 (9): 2209-2220.

19. Marberger M, Wilson T, Rittmaster R (2011): Low serum testosterone levels are poor predictors of sexual dysfunction. BJU Int., 108 (2): 256-262.

20. O'Connor D, Lee $\mathrm{D}$, Corona G et al. (2011): The relationships between sex hormones and sexual function in middle-aged and older European men. J Clin Endocrinol Metab., 96: 1577-87.

21. Cartagena-Ramos D, Fuentealba-Torres M, Rebustini F et al. (2018): Systematic review of the psychometric properties of instruments to measure sexual desire. BMC Med Res Methodol., 18 (1): 109-112.
22. Yee A, Aik O, Sien Y et al. (2020): Factor Structure, Reliability, and Validity of the Malay version of the Sexual Desire Inventory-2 (SDI-2-BM) in a Sample of Lower Urinary Tract Symptoms (LUTS) and Healthy Individuals. Malaysian Journal of Psychiatry, 29 (2): 1-10.

23. Zhou S, Zhao M, Yang Y et al. (2020): Age-related changes in serum reproductive hormone levels and prevalence of androgen deficiency in Chinese communitydwelling middle-aged and aging men: Two cross-sectional studies in the same population. Medicine (Baltimore), 99 (1): 18605-12.

24. Cooper L, Page S, Amory J et al. (2015): Matsumoto AM. The association of obesity with sex hormone-binding globulin is stronger than the association with ageing-implications for the interpretation of total testosterone measurements. Clin Endocrinol (Oxf), 83 (6): 828-833.

25. Liu C, Huang S, Cheng K et al. (2017): Lower SHBG level is associated with higher leptin and lower adiponectin levels as well as metabolic syndrome, independent of testosterone. Sci Rep., 7 (1): 2727-32.

26. Wu F, Tajar A, Pye S et al. (2008): Hypothalamicpituitary-testicular axis disruptions in older men are differentially linked to age and modifiable risk factors: the European Male Aging Study. Journal of Clinical Endocrinology and Metabolism, 93 (7): 2737-45.

27. Petering R, Brooks N (2017): Testosterone Therapy: Review of Clinical Applications. Am Fam Physician, 96 (7): 441-449.

28. Castelló-Porcar A, Martínez-Jabaloyas J (2016): Testosterone/ estradiol ratio, is it useful in the diagnosis of erectile dysfunction and low sexual desire? The Aging Male, 19 (4): 254-258.

29. Weiss R, Hohl A, Athayde A et al. (2019): Testosterone therapy for women with low sexual desire: a position statement from the Brazilian Society of Endocrinology and Metabolism. Archives of Endocrinology and Metabolism, 63 (3): 190-198.

30. Kang J, Ham B, Oh M et al. (2011): Correlation between serum total testosterone and the AMS and IIEF questionnaires in patients with erectile dysfunction with testosterone deficiency syndrome. Korean J Urol., 52 (6): 416-420.

31. O'Connor D, Lee D, Corona G et al. (2011): The relationships between sex hormones and sexual function in middle-aged and older European men. J Clin Endocrinol Metab., 96: 1577-87.

32. Chen T, Wu F, Wang X et al. (2020): Different levels of estradiol are correlated with sexual dysfunction in adult men. Sci Rep., 10: 12660-5.

33. Tan R, Cook K, Reilly W (2015): High estrogen in men after injectable testosterone therapy: the low $\mathrm{T}$ experience. Am J Mens Health, 9: 229-34. 